\title{
Perspective
}

PERSPECTIVE Actualité en histoire de l'art

1 | 2018

Actualité en histoire de l'art

\section{Anachronisme et interprétation : l'historiographie de Jean Jacques Lequeu}

Anachronism and Interpretation: the Historiography of Jean Jacques Lequeu

Anachronismus und Interpretation: Historiographie von Jean Jacques Lequeu

Anacronismo e interpretazione: la storiografia di Jean Jacques Lequeu

Anacronismo e interpretación: la historiografía de Jean Jacques Lequeu

Laurent Baridon, Jean-Philippe Garric et Martial Guédron

\section{OpenEdition}

Journals

Édition électronique

URL : http://journals.openedition.org/perspective/9493

DOI : $10.4000 /$ perspective. 9493

ISSN : 2269-7721

Éditeur

Institut national d'histoire de l'art

Édition imprimée

Date de publication : 30 juin 2018

Pagination : 129-144

ISBN : 978-2-917902-46-2

ISSN : 1777-7852

\section{Référence électronique}

Laurent Baridon, Jean-Philippe Garric et Martial Guédron, « Anachronisme et interprétation

I'historiographie de Jean Jacques Lequeu », Perspective [En ligne], 1 | 2018, mis en ligne le 31

décembre 2018, consulté le 01 octobre 2020. URL : http://journals.openedition.org/perspective/9493 ; DOI : https://doi.org/10.4000/perspective.9493 
Laurent Baridon, Jean-Philippe Garric, Martial Guédron

\section{Anachronisme et interprétation : I'historiographie de Jean Jacques Lequeu}

L'œuvre de Lequeu (1757-1826), découverte au milieu du XXe siècle en raison des affinités qu'Emil Kaufman voulut y voir avec ses propres engagements (KAUFMANN, 1952), ne se rattache ni à une école ni à un mouvement et son époque l'a passée sous silence. Longtemps, elle resta ainsi un objet flottant, à peine situé, et d'autant plus propice aux interprétations contemporaines que l'histoire de l'architecture, se contentant de prendre acte de la singularité des dessins et du personnage, le laissait à part. Mais les travaux de ces trente dernières années, en élargissant le domaine de l'histoire de l'architecture à des thématiques, des groupes d'acteurs ou des figures en marge, en approfondissant l'étude de voies parallèles à celles de l'édifice, qu'il s'agisse de l'imprimé ou de l'enseignement, éclairent notre objet d'un jour différent ${ }^{1}$.

Devant cette connaissance à la fois plus fine et plus large des mondes de l'art, de l'architecture et de l'artisanat en France durant la période révolutionnaire, l'équipe réunie pour la préparation de l'exposition Jean Jacques Lequeu. Bâtisseur de fantasmes' ${ }^{2}$ était confrontée à un basculement. Il s'agissait de comprendre désormais la singularité de l'intéressé avec ce qui, en profondeur, le relie à son temps. Cela revenait à rompre avec une tradition interprétative, multiple certes, mais marquée par la constante de l'anachronisme, toujours attentive aux effets de miroirs vis-à-vis des questions contemporaines, mais beaucoup moins à des points pourtant aussi déterminants, dans cette époque de changements rapides et brutaux, que son parcours social, sa culture visuelle disséminée ou sa micro chronologie. Un tel choix, fondé aussi sur le constat d'une déconcertante série d'interprétations non seulement abusives mais inopérantes méritait une explication. Sans prétendre ici détailler les résultats d'un travail présenté par ailleurs ${ }^{3}$, il nous a paru nécessaire de soumettre au débat ce parti d'une césure avec les travaux précédents.

Jean Jacques Lequeu (1757-1826), en léguant son œuvre à la Bibliothèque nationale, attendait sans doute de la postérité la reconnaissance qu'il n'avait pas obtenue de ses contemporains. Bien qu'il ait construit quelques édifices et que quelques-uns de ses dessins aient été reproduits dans les recueils gravés de Jean Charles Krafft ${ }^{4}$, son nom est passé aux

Laurent Baridon, professeur d'histoire de l'art contemporain à l'université Lumière Lyon 2, travaille sur les imaginaires scientifiques et sociaux des artistes. Il s'intéresse plus particulièrement à l'architecture et à la satire graphique au XIXe siècle.

Jean-Philippe Garric, architecte et historien, professeur d'histoire de l'art contemporain à l'université Paris 1 - PanthéonSorbonne est spécialiste de I'histoire de l'architecture en France et en Europe de la fin de l'Ancien Régime à la Première Guerre mondiale.

Martial Guédron est professeur d'histoire de l'art à l'université de Strasbourg. Il étudie les liens entre sciences et arts à travers les représentations du corps humain, que ce soit du côté de l'idéalisation, que ce soit du côté du grotesque et du monstrueux. 
1. Jean Jacques Lequeu, "Sépulchre de l'auteur, frère de Jésus", dans Architecture civile, 1777-1825, pl. 33, fig. 100*, Paris, BnF, département des Estampes et de la Photographie, RESERVE FOL-HA-80 (1).

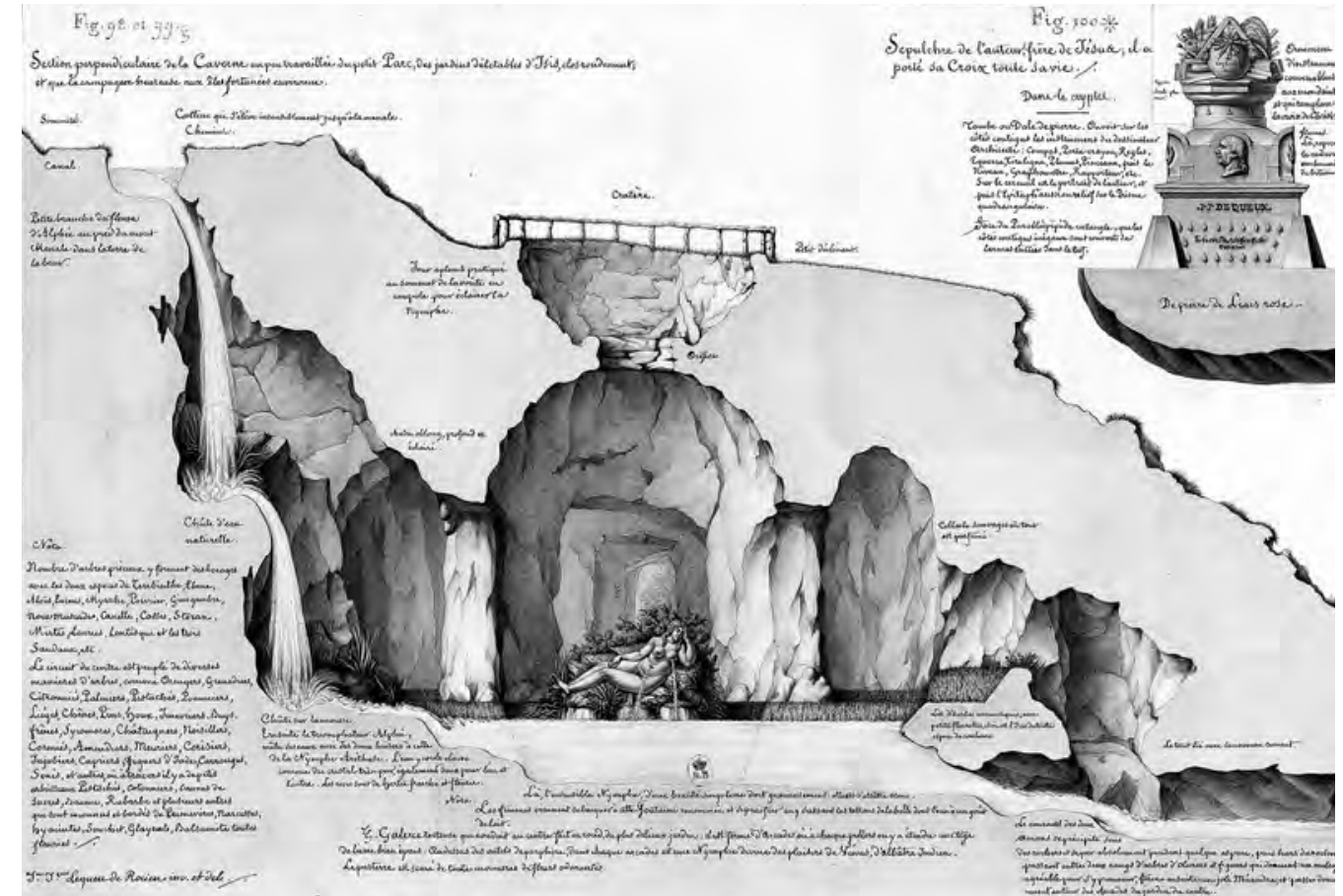

oubliettes de l'histoire pendant près d'un siècle. Peu de choses sont connues de sa vie. Les dessins qu'il a légués à la Bibliothèque nationale de France présentent des problèmes de datation. Leur qualité inégale pourrait même laisser supposer l'intervention de plusieurs mains. Le fonds Lequeu est hétérogène, comptant des documents techniques, des méthodes graphiques, des projets d'architecture, des scènes de genre et des dessins anatomiques dont certains clairement sexuels - les " figures lascives".

Lequeu est d'emblée anachronique, puisque la compréhension de son œuvre ne s'appuie pas sur une lignée bibliographique qui nous relierait à lui. Elle ne peut s'inscrire dans une historiographie constituée des commentaires ou des critiques émis de son vivant, de nécrologies, d'études monographiques ou générales, de catalogues de vente, d'expositions, de comparaisons, etc. Il a surgi du néant, comme quelques rares autres artistes. Vermeer est un de ceux-là et son œuvre, depuis sa redécouverte, a suscité une abondante et très diverse littérature ; l'absence de sources fiables et de tradition interprétative excite l'imagination des exégètes.

Mort en 1826, resté dans une large mesure invisible à son époque, «inventé » du tout au tout par le $\mathrm{XX}^{\mathrm{e}}$ siècle en raison d'une production graphique qui fascine, Lequeu ressuscite en 1933 quand Emil Kaufmann exhume une partie de son œuvre à la Bibliothèque nationale de France. Dix-sept ans plus tard, il devient un artiste "majeur " - avec la découverte des "figures lascives " et des portraits, signalés par Helen Rosenau (RosenAU, 1950). Or il se prête d'autant plus aisément aux interprétations audacieuses - pour ne pas dire fallacieuses qu'on ne dispose guère le concernant des précieuses sources historiques traditionnelles, que son ouvre ne s'accompagne d'aucune tradition critique et qu'on ne peut mobiliser à son propos que de très rares éléments d'évaluation contemporains, sinon le silence dont il fait l'objet. Ce n'est pas qu'il soit muet sur lui-même, car il est prolixe, au contraire, de notes et de commentaires manuscrits (fig. 1), mais la forme de ces écrits imparfaits - parce que mal orthographiés, utilisant une syntaxe erratique, semblant parfois incohérents et témoignant de façon plus générale d'une maîtrise de la langue qui n'est pas d'un homme éduqué -, 
les a disqualifiés aux yeux d'historiens habitués à autre chose, au point que leur authenticité a été mise en cause. Ainsi, balloté par une historiographie qui le présente tour à tour comme un moderniste, un surréaliste, un postmoderne et finalement un déséquilibré, le personnage, comme son ouvre, s'obscurcissent à mesure que les travaux le concernant se superposent, pour demeurer in fine une " énigme architecturale " selon le titre aporétique de la première et de la seule monographie qui lui fut consacrée en 1987 par Philippe Duboÿ : on y trouve une interprétation audacieuse de l'œuvre, qu'il est suggéré de regarder au prisme d'une autre, celle de Marcel Duchamp. Cette démarche part de l'hypothèse selon laquelle Duchamp aurait connu les dessins de Lequeu bien avant Emil Kaufmann. L'auteur rapporte par ailleurs que Teeny Duchamp, la veuve de l'artiste, lui a déclaré : " Mon mari avait deux passions, Raymond Roussel qu'il a vu et Jean Jacques Lequeu qu'il n'a jamais connu » (DUBOŸ, 1987, p. 352). La question qui l'intéresse n'est pas de savoir si Duchamp a pu être marqué par la découverte des dessins de Lequeu au point qu'ils auraient pu déterminer une partie de son œuvre. Il s'agit bien d'interpréter Lequeu à la lumière de Duchamp et non l'inverse.

\section{Lequeu anti-classique?}

L'historiographie du sujet montre que l'étude de Lequeu a toujours suscité beaucoup de questions chez ceux qui l'ont approché. Les rares mentions dont il a fait l'objet, par Henri Bouchot et par Charles Lucas, sont assez sommaires 5 . Parmi les architectes "indépendants", "symbolistes " et "bizarres ", Lequeu est surtout repéré comme "le plus curieux de tous $^{6}$ ". Pour autant, les premières analyses de l'œuvre ne relèvent pas d'une interprétation anachroniste. En 1933, Emil Kaufmann mentionne son travail dans son premier livre important, Von Ledoux bis Le Corbusier, en concluant : " une œuvre très intéressante qui ouvre de nouveaux espaces à l'histoire de l'art... Déjà 150 ans avant l'architecture moderne le programme de la Sachlichkeit apparaissait ${ }^{7}$. " On reconnaît ici la thèse principale de ce livre célèbre : le développement de l'architecture " autonome " du mouvement moderne aurait ses origines dans les volumétries fortes et simples du " néoclassicisme ". En 1952, dans "Three Revolutionary Architects ", après la découverte de l'ensemble des " figures lascives ", il réitérait son point de vue en l'explicitant :
Ses fantaisies bizarres révèlent beaucoup de son époque pour qui s'intéresse au développement des idées artistiques plutôt qu'aux progrès pratiques. Bien que Lequeu, comme tous les Romantiques, regardait en arrière vers le passé et des régions lointaines, il était néanmoins le précurseur d'un courant important du premier XX' siècle. Aux deux périodes, de mêmes mouvements et de mêmes incertitudes inspirent d'étranges productions; aux deux périodes l'expression compte davantage que le formalisme; ainsi, de nouvelles auvres, grandioses et dignes, émergent de la tourmente. Il n'est pas dans mon intention de sous-entendre des liens directs entre 1800 et 1900. Je ne me préoccupe que d'une continuité des idéess.

Si, pour Kaufmann, Lequeu est le promoteur d'une conception moderniste de l'architecture à la fois autonome, anachronique et autotélique - autrement dit n'ayant pas d'autre véritable finalité qu'elle-même ${ }^{9}$-, cette continuité des idées n'exclut pas qu'il dépend de son contexte historique :

Je pense que c'est moins sa condition personnelle que le mouvement général de son époque qui permet en premier lieu de rendre compte de sa production [...] Bien que Lequeu divague bien au-delà des limites fixées par les règles, ses fantaisies sont davantage que des extravagances. Ce sont des auvres d'art qui permettent de comprendre l'homme et au-delà d'appréhender son époque ${ }^{10}$. 
Dès l'introduction, il affirme que Lequeu compte parmi les " trois architectes révolutionnaires " parce que son œuvre correspond à un moment de cette période : "Des trois, Boullée représente surtout la lutte pour les formes nouvelles; Ledoux la recherche d'une nouvelle composition des éléments ; Lequeu l'ultime et tragique étape du mouvement révolutionnaire - désespoir, résignation et retour au passé1 ${ }^{1}$. " Ainsi Lequeu est à la fois reconnu pour sa position spécifique et assimilé à deux architectes dont la recherche d'une nouvelle architecture est comparable à celle des avant-gardes modernes.

En 1949, Helen Rosenau a contribué à cette comparaison de Lequeu avec Boullée et Ledoux, établissant un certain nombre de convergences ${ }^{12}$. Dans un postscript paru en 1950, deux ans avant les " Three Revolutionary Architects " de Kaufmann, elle fait part de la découverte des portraits et des "figures lascives". Dans ce court texte, elle propose des comparaisons possibles avec Füssli ou Diderot et indique qu'il faut peut-être considérer Lequeu comme un écrivain, autant que comme un architecte et un artiste ${ }^{13}$. Or cette interrogation sur ce qui lie Lequeu à son époque est restée lettre morte.

Une autre voie a été privilégiée dans un premier temps pour interpréter son ouvre: celle de la périodisation stylistique. Louis Hautecoeur, bien que tout son engagement soit tourné, à l'inverse de Kaufmann, vers une défense de la continuité contemporaine du classique $^{14}$, partage cependant avec ce dernier la conviction d'une rupture autour de 1900. Il justifie cette limite chronologique de sa monumentale Histoire de l'architecture classique en France par le postulat que «Le XVI siècle, en son principe, ressemble au XXe à ses débuts : un monde achève de mourir, un autre apparaît à la vie. Croyances, institutions, sciences, arts, tout est remis en question, tout est animé d'un esprit nouveau ${ }^{15}$. " Dans le cinquième tome de cet ouvrage, reprenant presque mot pour mot Kaufmann sans le citer, il rattache Lequeu au "romantisme, épris des civilisations passées et lointaines ", comme Boullée, Poyet ou Desprez, jugeant qu'il annonce "l'éclectisme du XIX siècle $^{16}$ ". Sans accorder à la fin du siècle des Lumières le même rôle charnière qu'un Georg Germann ${ }^{17}$, il voit cependant en Lequeu l'un des fossoyeurs de la grande tradition classique.

Dix ans plus tard, tout en partant du même postulat, Jacques Guillerme aborde l'œuvre de Lequeu en inaugurant une série d'études relevant de l'anachronisme ${ }^{18}$. Qualifiée de " [s]ource pure du mauvais goût architectural de la seconde moitié du XIXe siècle " (GUILLERME, 1965, p. 153-154), elle est caractérisée de façon plus nuancée quelques années plus tard " entre l'irrégulier et l'éclectique » (GuILLERME, 1974). Le Rendez-vous de Bellevue (fig. $\mathbf{2}$ et $\mathbf{3}$ ) est le sujet de cette seconde étude, projet qu'Emil Kaufmann, en 1952, avait déjà décrit comme un objet hésitant entre deux attitudes : "Mélange d'incohérences et d'incongruités, le Rendez-vous de Bellevue en dit long sur cette période qui lutte pour l'innovation et qui toujours et toujours se tourne vers un passé hanté de rêves étranges ${ }^{19}$. " Mais ce qui, chez Kaufmann, était caractéristique du tournant des années 1800, devient chez Guillerme une forme d'incertitude chronologique et stylistique. Cette attitude participe d'une remise en cause des cadres traditionnels de la périodisation et de la classification stylistique. Les originalités et les étrangetés de Lequeu constituent un cas idéal pour démontrer leur caducité.

Il revient à Philippe Duboÿ d'avoir donné à cette démarche un tour plus radical, construisant son approche dans une série d'articles à partir de 1974 jusqu'à la publication de sa monographie en 1987. Signe des temps, dans sa contribution au dossier spécial que Les Cahiers du musée national d'art moderne consacraient, en cette même année, à l'après-modernisme, Thomas McEvilley pouvait écrire la chose suivante : "L’Histoire ne paraît plus avoir de forme générale, ni se diriger vers un point précis. Elle se dissout dans le temps, dans cette succession sans fin de moments, décrite par Averroès, sans orientation ni forme globale ${ }^{20}$. " 


\section{Lequeu postmoderne?}

Philippe Duboÿ a projeté sur l'œuvre de Lequeu un point de vue implicitement et ironiquement moderne, mais involontairement postmoderne par sa subjectivité, son relativisme et sa forme même : dissémination, esthétique du collage, de la citation détournée ou réinvestie, goût du brouillage ludique des références et des repères. Désormais, le Lequeu désemparé, tourné vers le passé dont Kaufmann nous entretient est pris dans la logique de la modernité, celle qui n'opérerait que par ruptures, en opposition avec ce qui reste tributaire de la tradition. Si, très vite, il s'est trouvé aiguillé vers l'hybride et l'impureté, avec Duboÿ, il est devenu une sorte de fake. Avec l'œuvre de Duchamp, le voici abordé par le biais d'un montage qui le lie ponctuellement à Jacques Lacan, à l'utopie de Charles Fourier ou, comme dans la citation suivante, au Raymond Roussel de Michel Foucault :

\section{COMMENT J'AI ÉCRIT CERTAINES DE MES ARCHITECTURES! Les constructions littéraires, les jeux de paroles, les calembours phonétiques, armes favorites de Lequeu, sont une référence précise à l'écriture "blanche et lisse " de Raymond Roussel; celle de Lequeu est "Blanche et occulte" mais aussi lyssée (Royale, avec des fleurs de lys) ${ }^{21}$.}

Par ailleurs, le Lequeu dépeint par Duboÿ procède d'une historiographie psychopathologique qui a été appliquée à de nombreux artistes. Or le tableau clinique de l'intéressé n'a cessé de s'aggraver au fil des redécouvertes dont il a fait l'objet. Sobrement signalé de son vivant comme architecte parisien dans le dictionnaire des artistes de Johann Rudolf et Johann Heinrich Füssli²2, il est doté d'une imagination " un peu maladive ${ }^{23}$ " dès la fin du XIX ${ }^{\mathrm{e}}$ siècle. Ensuite, on a le sentiment que sa santé mentale n'a plus cessé de s'altérer, au point que certains auteurs le voient finir ses jours dans un $a^{2}$ sile $^{24}$. Auparavant son cas aura été examiné à travers le prisme de la psychiatrie et de la psychanalyse, tout en se trouvant affilié, a posteriori, aux "Surréalistes malgré eux ${ }^{25}$ ", sans qu'aucun des membres fondateurs du groupe n'ait jamais fait état d'un intérêt particulier pour lui, sans que ceux qui ont suggéré de tels liens ne nous expliquent jamais en quoi

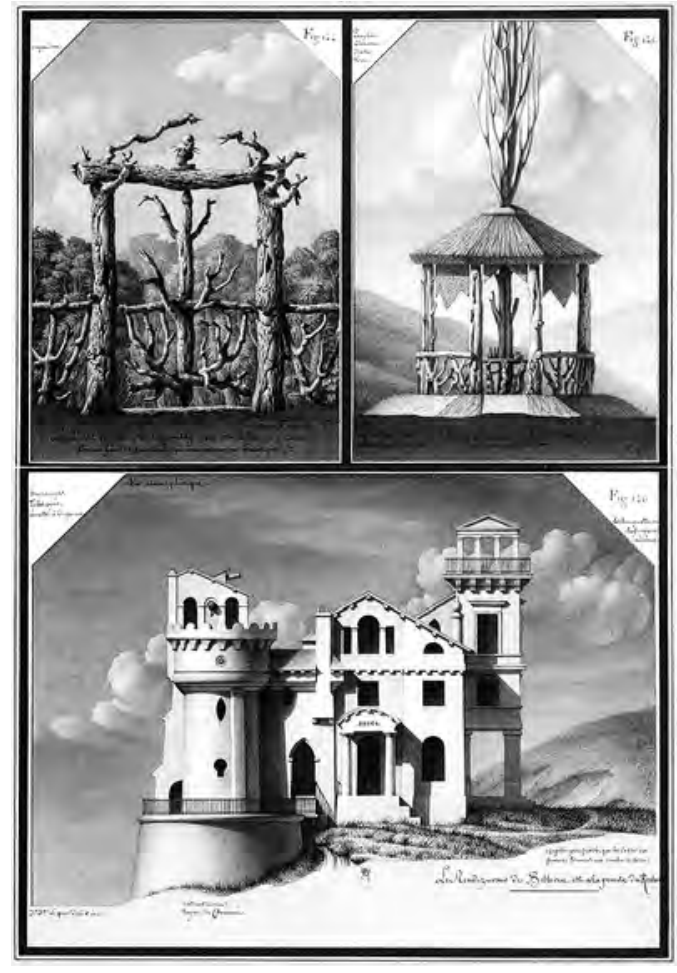

2. Jean Jacques Lequeu, " La porte de clôture de l'hermitage est sur le chemin solitaire comme pour les anachorètes qui menaient une vie érémitique : Vide-bouteille du desert aride ;

Le rendez-vous de Bellevue est à la pointe du rocher », dans Architecture civile, 1777-1825, pl. 55, fig. 144, 145 et 146, Paris, $\mathrm{BnF}$, département des Estampes et de la Photographie, RESERVE FOL-HA-80 (2).

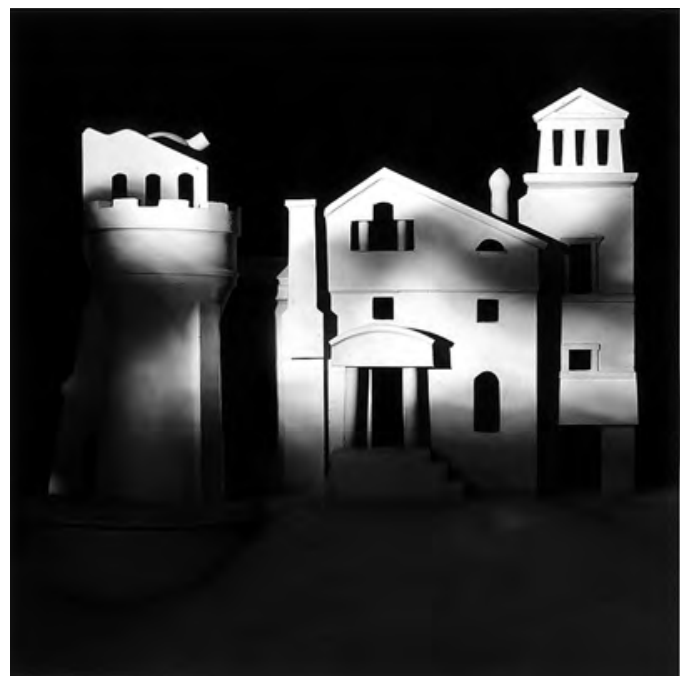

3 James Casebere, Rendez-vous de Bellevue, 1991, Frac centre. 
la production de cet architecte dessinateur répond à la définition qu'André Breton a donnée de l'image surréaliste ${ }^{26}$. Un parallèle peut être proposé avec l'historiographie de Grandville, également marquée par un prisme psychopathologique. Elle est discutée par Laure Garcin, à une époque proche de celle des travaux de Duboÿ, et dans une démarche comparable, puisqu'elle propose de considérer Grandville à la lumière du surréalisme et de la psychanalyse ${ }^{27}$. Tout en usant volontiers d'anachronismes, elle s'en distingue radicalement dans la mesure où elle se fonde sur un intérêt effectif des surréalistes et surtout de Breton pour l'illustrateur nancéien : "Surréaliste, il l'a été bien avant la création de ce mot, et bien avant que ne fussent établies les méthodes psychanalytiques dont les surréalistes se recommandent si souvent ; car il avait deviné par intuition l'importance du phénomène du rêve en tant que moyen de connaissance du moi des profondeurs $»^{28}$.

Rappelons que lorsque Charles Jenks popularise, en 1977, la notion de postmodernisme appliquée à l'architecture, il explique aussi que la première utilisation du mot " postmoderne " relativement à l'architecture est apparue dès 1949, aux prémices de la crise du mouvement moderne, soit au moment même où Emil Kaufmann consacrait à Lequeu son article fondateur (KAUFMANN, 1949). Cette réaction contre la doxa moderniste s'exprime par le refus d'une architecture qui fait du bâtiment une sorte de sculpture monumentale, mais aussi à travers une propension à l'ostentation, à l'hétérogénéité des styles, à de multiples références au passé et à la culture populaire. Ce qui est bien souligné dans l'ouvrage canonique des Venturi : "Cependant, ce n'est pas une innovation que d'approfondir sa compréhension à partir du banal : les beaux-arts prennent souvent la relève des arts populaires. Les architectes romantiques du dix-huitième siècle redécouvrirent une architecture rustique conventionnelle qui existait ${ }^{29}$. " Au passage, il est tentant de se demander dans quelle catégorie les Venturi auraient rangé les fabriques de Lequeu : la spectaculaire étable-vache semble se situer dans la famille des bâtiments devenus enseignes, sur le modèle de l'édifice-canard dont la forme évoque l'usage (fig. 4 et 5), mais son belvédère ou Temple de la Nature dans celle des " hangars décorés" (decorated sheds), autrement dit ces édifices montrant des signes symboliques qui en explicitent la fonction.

Quoi qu'il en soit, la réception critique de Lequeu montre qu'une certaine vision puriste de l'architecture moderniste a pu servir de contrepoint à la lecture de son ouvre et que celle-ci a parfois été vue au filtre de l'éclectisme du XIXe siècle, voire du postmoderne. Kaufmann relève le caractère hétérogène des sources d'inspiration de Lequeu, ce qui, pour lui, aboutit

4. Jean Jacques Lequeu, "L'étable à vache tournée au midi est sur la fraîche prairie ", dans Architecture civile, pl. 74, fig. 174, Paris, $\mathrm{BnF}$, département des Estampes et de la Photographie, RESERVE FOL-HA-80 (2)

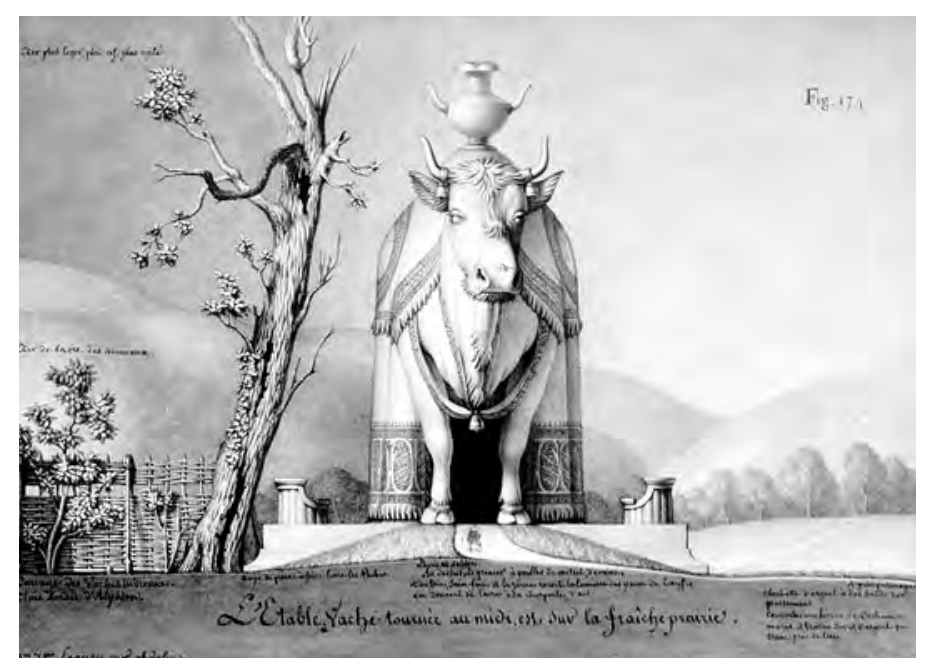
à une confusion des styles empruntés à divers pays et diverses époques : " Il y a une maison égyptienne, une maison chinoise, une maison turque, une petite synagogue, un porche et un sanctuaires persans, un poulailler aux motifs orientaux, un pressoir et un pigeonnier, mélanges de formes orientales et géométriques, ainsi que différents projets gothicisants comme la laiterie ou la façade du Temple d'Isis ${ }^{30}$. " Dès 1965, dans la Gazette des Beaux-Arts, Jacques 


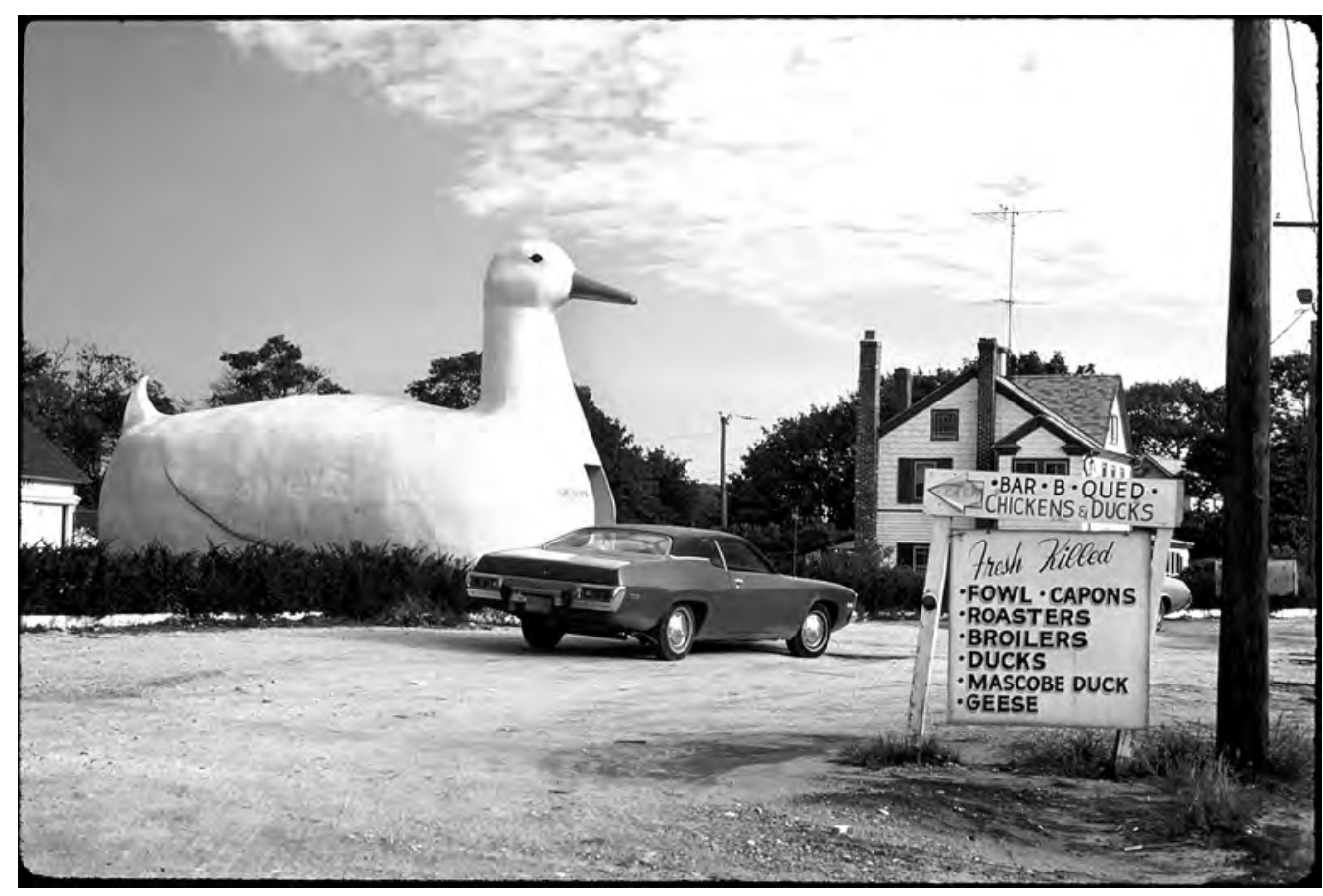

5. Le drive-in en forme de canard reproduit par Robert Venturi et Denise Scott Brown, dans Learning From Las Vegas, Cambridge, MIT Press, 1977

Guillerme considère déjà cet " éclectisme " comme un trait fondamental du "mauvais goût " de Lequeu, tout en le reliant à sa " voracité encyclopédique ${ }^{31}$ ». Or le ton change assez nettement au cours de la décennie suivante, quand s'affirme la question essentielle des intentions exactes et délibérées qu'il est permis de prêter à Lequeu ; entre temps, la plupart des théoriciens du postmodernisme, au-delà de leur diversité, ont observé que ce courant favorise la parodie, le pastiche et le simulacre ${ }^{32}$.

Dans un nouvel article qu'il lui consacre en 1974, Jacques Guillerme interprète plusieurs dessins de Lequeu comme des "calembours plastiques ". Dans l'un d'eux, il remarque que Priape se montre sous la forme d'un terme couronné d'une tête de dogue dont le nez est formé, comme le souligne une inscription, d'un "membre épouvantable " : « [...] le mélange des genres et des thèmes, note Guillerme, est chez Lequeu très résolument composé, tout en étant marqué d'une évidente intention burlesque ${ }^{33}$ ". Désormais, même si la fonction cathartique de sa production graphique reste prise en compte, l'irrégularité et l'éclectisme de Lequeu apparaissent moins comme l'expression d'une déréliction mentale que d'une dérision parodique, une manière de maintenir sa différence et de prendre ses distances face au dogmatisme des architectes révolutionnaires. Cinq ans plus tard, le même Jacques Guillerme enfonce le clou : Lequeu est doté d'un " génie parodique » qui « s'exerce sur l'œuvre construit, figuré ou écrit de ses contemporains ${ }^{34} "$.

Dans l'intervalle, la grande entreprise de projection rétroactive opérée par Duboÿ a trouvé son accroche : l'affiliation de Lequeu à Duchamp ${ }^{35}$, à la faveur de certaines stratégies d'appropriation qui tentent d'associer Duchamp au postmodernisme. Le milieu des années 1970 consacre en effet la redécouverte de l'artiste en France. Elle est notamment marquée par la publication, en 1975, de notes, d'aphorismes et d'articles divers au contenu souvent sibyllin ${ }^{36}$ qui ont pu encourager la promotion de l'ambiguïté, de l'indétermination et d'un scepticisme joueur au rang de catégories de pensée propres à mieux cerner l'époque ${ }^{37}$. Une dizaine d'années plus tard, l'idée que le père des ready made, avec la complicité de Georges Bataille, 


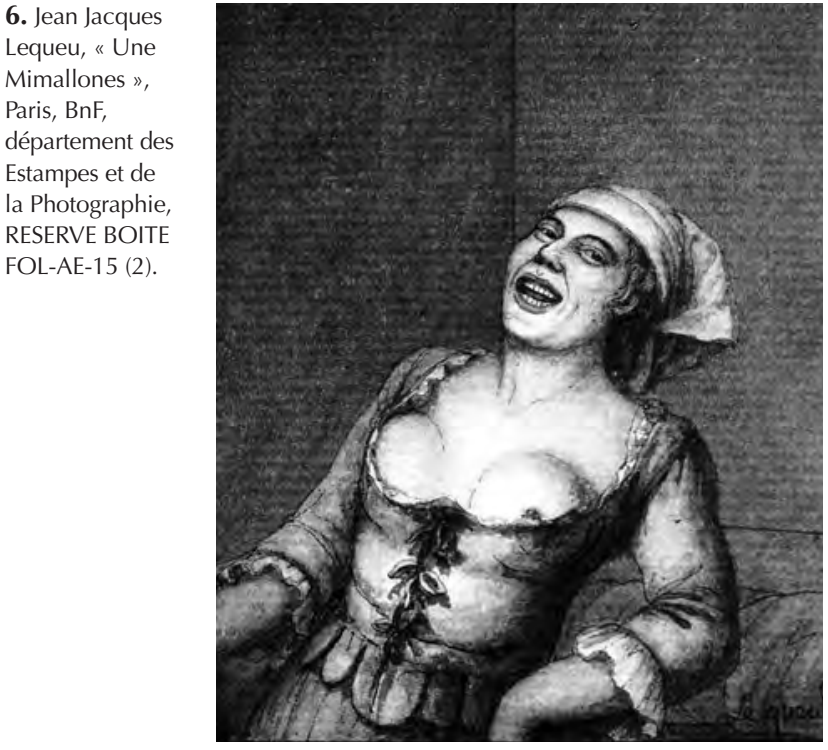

serait largement intervenu dans les dessins que Lequeu a légués à la Bibliothèque nationale, au point d'en faire une œuvre de Duchamp dirigée contre Le Corbusier, est au cour de la monographie de Duboÿ, publiée en 1987, soit au moment du centenaire des deux intéressés ${ }^{38}$.

Les propos de Jean Nouvel, dans son dialogue avec Jean Baudrillard, contribuent à comprendre la démarche de Duboÿ : " On peut se demander pourquoi il n'y a pas l'équivalent de Duchamp dans le monde de l'architecture. Il n'y a pas d'équivalent parce qu'il n'y a pas d'autoarchitecture. Il n'y a pas d'architecte qui puisse être un geste scandaleux, immédiat, accepté. Des architectes ont essayé de se heurter à ces limites-là. Ce fut le départ de la postmodernité ${ }^{39}$. " Lequeu, cela tombe plutôt bien, n'a rien laissé de bâti ; quand on privilégie un parti pris textuel sophistiqué qui tend à dématérialiser l'architecture ${ }^{40}$, il a l'avantage d'offrir un cas exemplaire. Mais on conserve de lui toute une production graphique à travers laquelle ses exégètes n'ont cessé de vouloir remonter de l'œuvre à l'homme, au point de confondre, dans tous les sens du terme, ses autoportraits, ses études de têtes d'expression, ses figures lascives, ses fabriques, ses projets d'édifices privés et publics, ses fantaisies architecturales, etc., au prix d'anachronismes d'autant plus flagrants qu'ils sont activés par une tendance à rejeter le modèle historique d'engendrement du sens. Comme si l'abandon des grands récits ${ }^{41}$ et la réévaluation nécessaire de l'histoire comme progrès linéaire légitimaient, dans tous les cas de figure, de substituer l'intention à la réalisation et l'essence à l'historicité.

Dans Le postmodernisme ou la logique culturelle du capitalisme tardif, Fredric Jameson affirme que le postmodernisme a mis à mal la conception moderniste du style en tant qu'expression unique et personnelle du sujet individuel, avec pour conséquence un rapport particulier au passé : "Car avec l'effondrement de l'individu haut-moderniste du style [...] les producteurs culturels ne peuvent plus se tourner vers autre chose que le passé : l'imitation des styles morts... " Pour Jameson, cette situation a favorisé l' " historicisme ", autrement dit " la cannibalisation aveugle de tous les styles du passé, le jeu de l'allusion stylistique aléatoire, et, de façon générale, le primat croissant de ce qu'Henri Lefebvre a appelé le "néo" 42 ". C'est également l'avis de David Kolb, selon qui le postmodernisme, à la parodie, combine la distanciation ironique, l'autoréférence et la transgression des frontières séparant les styles, les idiomes et les genres ${ }^{43}$. Aussi, comme l'explique David Harvey, l'éclectisme postmoderne est-il indissociable d'un rapport particulier à la temporalité : tout en évitant les notions de progrès et de continuité historique, tout en tenant la mémoire à distance, ses adeptes développent une étonnante capacité à piller le passé et à incorporer tout ce qu'ils y trouvent avec certains aspects du présent ${ }^{44}$. Lyotard observe en ce sens que cet éclectisme permet de diluer les traits saillants des styles auxquels il emprunte et que la temporalité paradoxale du postmoderne est celle du futur antérieur ${ }^{45}$.

Autant dire qu'il est tentant d'historiciser les arguments des uns et des autres à propos de Lequeu, surtout quand les commentateurs se laissent aller à des généralisations anhistoriques. Prenons, par exemple, le sort réservé au soi-disant autoportrait en travesti (fig. 6), qui a occasionné des interprétations sur le modèle des gender crossing, ces jeux sur les identités sexuelles dont Claude Cahun et Marcel Duchamp, au début des années 1920, avaient été 
les pionniers ${ }^{46}$. Voir dans ce dessin médiocre un « autoportrait " sans en fournir la moindre justification est déjà problématique, mais en conditionner la lecture à travers la photographie de Man Ray montrant Duchamp sous l'aspect de son alter ego féminin Rrose Sélavy l'est plus encore, comme s'il était possible de mettre sur le même plan un artiste qui s'est ingénié à brouiller son image publique tandis que sa renommée ne cessait de croître et un autre dont la quête de reconnaissance est demeurée jusqu'à la fin inopérante. Il serait sans doute plus utile de montrer en quoi Lequeu, par ses autoportraits avérés (fig. 7), s'inscrit dans des pratiques historiquement déterminées, reposant sur toute la tradition qui, au moins depuis le XVIII ${ }^{\mathrm{e}}$ siècle, croise l'autoreprésentation et le jeu de rôle.

\section{Lequeu anachronique?}

Lequeu est particulièrement pertinent pour prolonger la réflexion sur l'anachronisme. Georges Didi-Huberman a mis en critique

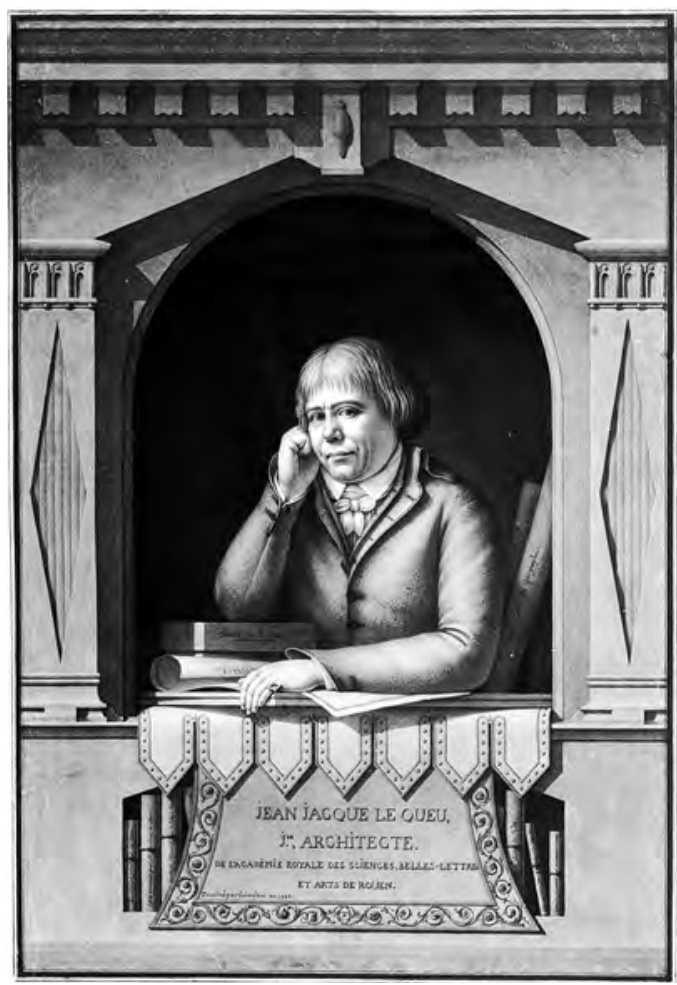
ce qui fonde l'éthique de l'objectivité historienne de l'histoire de l'art : le lien synchronique de l'œuvre à ses contextes. Selon l'auteur de Devant le temps (DIDI-HuBERMAN, 2000), il est vain de prétendre retrouver l'esprit et le regard d'une époque en étudiant une œuvre d'art. Nous ne pouvons en avoir qu'une compréhension différée. Il est impossible de ne pas être anachronique, sauf à n'être qu'un faiseur de chroniques, c'est-à-dire n'énoncer que des faits contemporains de celui qui les écrit - encore les décrit-il toujours a posteriori, d'après sa propre perception et dans un après-coup. Selon Georges Didi-Huberman, c'est cette anachronicité même qui doit focaliser l'attention de l'exégète et non l'espoir de la juguler.

C'est ainsi qu'Hubert Damisch - reprenant une phrase de Walter Benjamin sur la littérature $^{47}$ - recommande à propos des œeuvres, "dans le temps où elles sont nées, de présenter le temps qui les connaît ${ }^{48} "$; il revendique de les interpréter de façon à comprendre leurs " effets au-delà du moment qui les a vus naître ". Il s'agit de "rendre compte des temporalités complexes qui traversent chaque ouvre et la lient à d'autres à travers l'histoire, composant des constellations, plus ou moins grandes, dont le montage peut relever du travail d'un artiste autant que de celui d'un historien de l'art" ${ }^{49}$ ". Cette démarche revendique une forme de subjectivité qui peut s'exprimer par des "fictions théoriques", légitimées par le fait qu'il s'agit de comprendre les effets des œuvres sur un individu. Ne s'agit-il pas d'un déplacement des enjeux ?

Il fait peu de doute que presque tous les artistes se nourrissent d'anachronismes. Le plus important est celui qui fonde la Renaissance et toutes les renaissances identifiées comme telles par Panofsky. Alexander Nagel et Christopher S. Wood ont tenté de renouveler la question en proposant que la Renaissance italienne soit marquée du sceau d'un double rapport au temps (NAGEL, WOOD, 2010). L'un, qualifié de performatif, qui procède d'une conception traditionnelle et linéaire de l'historicité. L'autre, qualifié de substitutif, qui relève d'un lien de nature magique avec un original absent. Il a déjà été remarqué que cette catégorisation découlait des notions
7. Jean Jacques Lequeu, Autoportrait, dans Architecture civile, Frontispice, Paris, BnF, département des Estampes et de la Photographie, EST RESERVE HA-80 (1). 
d'image et de culte chez Hans Belting. L'attitude substitutive, qui ne relève pas de liens de causalité, est évidemment liée à l'anachronisme tel que Georges Didi-Huberman l'a défini, contre l'historicité traditionnelle de l'histoire de l'art. Dans Devant le temps, ce dernier fait du conflit entre Panofsky et Benjamin - autour de l'héritage de Warburg et de ses "survivances ", ce paradoxe fatal à une conception rectiligne de l'histoire ${ }^{50}$ l'événement fondateur du divorce entre une histoire de l'art académique et une autre qui peut se définir par des anachronismes heuristiques. Alors que Panofsky, avec l'iconologie, fondait une histoire expliquant l'art par ses contextes, Benjamin en appelait à une " histoire de l'art des prophéties ${ }^{51}$ ".

Il n'est pas anodin que les ouvres étudiées par l'un et par l'autre soient de nature très différente. Panofsky travaille presqu'exclusivement sur des périodes anciennes. Il n'a interrogé l'art de son temps que pour ramener la calandre de la Rolls-Royce à l'archétype de la façade de temple. Il en va différemment de Warburg et de Benjamin dont les intérêts sont chronologiquement beaucoup plus étendus. Au moment où les horizons géographiques et historiques s'ouvrent largement, Warburg recherche les parentés entre les Pathosformeln amérindiens et européens. Au moment où les productions culturelles se transforment sous l'effet de la reproductibilité, Benjamin interroge en " chiffonnier " les productions " fétichistes " de la marchandise comme un archéologue du présent. Dans les deux cas, leur réflexion désavoue les distances chronologiques, proclament une forme d'autonomie de l'œuvre d'art qui l'émancipe du temps historique. Mais, en ce sens, leur réflexion est bien du temps des avant-gardes modernes, parce qu'elle suppose une rupture ou au moins une anticipation qui la fait sortir de son époque. Rappelons qu'au même moment, dans l'entre-deux-guerres, Kaufmann propose également de lier Boullée, Ledoux et Lequeu dans une même origine de l'autonomie de l'architecture qui enjambe le XIX siècle " de Ledoux à Le Corbusier ».

Ce qui justifie cet anachronisme du regard de l'historien de l'art chez Kaufmann, c'est précisément que le rapport des architectes à leur époque est de même nature et que ces époques présentent également des parentés. Kaufmann n'utilise une approche anachronique que pour rendre compte de la relation de ces architectes à leurs époques respectives. Reste que cette attitude procède d'une démarche subjective d'autant plus forte que la relation même qui lie Kaufmann à son temps est particulière, comme elle l'est bien entendu pour Panofsky et Benjamin, avec des destins et des idées très différentes.

Il ne s'agit pas ici de réfuter le caractère vain et aporétique de l'objectivité absolue, ou de refuser d'envisager les écarts irréductibles qui nous séparent de la culture visuelle d'un Lequeu. Il ne s'agit pas non plus de contester le fait que cet artiste, comme tous ceux qui sont dignes d'intérêt, possède une dimension intempestive, dans le sens où elle révèle parfois des décalages avec l'évolution du goût. Enfin, il ne s'agit pas de nier que les confrontations d'œuvres chronologiquement éloignées sont susceptibles de s'éclairer l'une l'autre ${ }^{52}$. Cela n'obère en rien le fait qu'il convient d'interroger la nature de ses étrangetés par une approche franchement contextuelle en tirant parti de nos connaissances actuelles sur la période. Les caractéristiques graphiques et les trouvailles architecturales cultivent un goût de l'originalité que Lequeu n'était pas le seul à développer. Un rapprochement attentif de ses projets avec les publications contemporaines de Georges Louis Le Rouge ou de Jean Charles Krafft permet de mieux faire la part de sa dette vis-à-vis des productions de son époque et de la spécificité de ses propositions ${ }^{53}$. Le personnage lui-même, avec ses zones d'ombre, peut être aujourd'hui mieux cerné grâce aux travaux sur des figures d'artisans et d'artistes qui lui sont contemporaines et qui ont présenté les mêmes ambitions statutaires et sociales. Car une grande partie du fonds Lequeu, méconnue parce que souvent jugée ennuyeuse, est composée de dessins de machines, de dissertations techniques et d'innombrables dessins de charpente. De même, si ses obsessions sexuelles et anatomiques peuvent faire l'objet d'une approche psychanalytique, elles sont très repérables dans la culture du tournant des Lumières. Ainsi on pourra relever, 
chez lui, un goût pour les figures allégoriques inspirées de l'iconographie classique, qui, doublé d'une affection particulière pour les attributs de la fécondité et des amours, rappelle à la fois l'intérêt que ses contemporains portaient aux déesses nourricières de la mythologie et les formes amples des figures du calendrier révolutionnaire dessinées par Louis Lafitte et gravées par Salvatore Tresca. Bien entendu, les petites intrigues érotiques de Lequeu se rattachent aussi à la vogue des estampes licencieuses qui, à en croire Louis Sébastien Mercier, se sont alors multipliées le long des quais de la Seine et sur les boulevards, au risque d'alarmer la pudeur par des attitudes et des postures lascives ${ }^{54}$. Plus radicale, la manière dont il a pu focaliser sur le sexe féminin à travers ses dessins d'une grande crudité serait incompréhensible si l'on omettait de les rapporter à l'intérêt savant de la période pour le dimorphisme sexuel ${ }^{55}$ ainsi

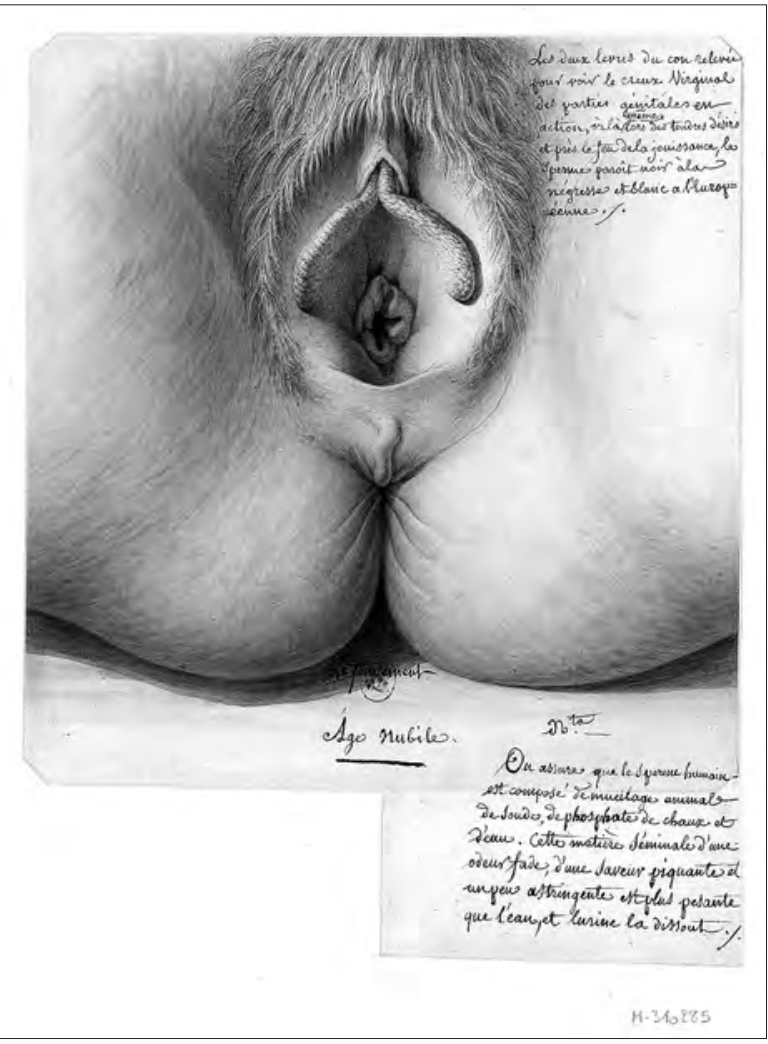
qu'à certaines planches anatomiques dont il s'est inspiré, notamment celles de Jacques Fabien Gautier d'Agoty pour son traité sur les maladies vénériennes ${ }^{56}$ (fig. 8). Les suggestives parentés entre dessins anatomiques et volumes architecturaux ressortissent à une lecture sexuelle et à une mise en crise de paradigmes de la théorie classique. Associer des femmes dénudées à des baies, comme il le fait au sein d'une série de représentations, relève de la théorie des caractères (fig. 9). Les obsessions pour les béances des grottes, apparentées aux séries de vulves, les phallus dressés comme autant de colonnes témoignent sans doute d'une érotomanie intéressante à diagnostiquer, mais peuvent également être rapprochés des recherches historico-érotiques sur le culte de Priape qui marquent la fin du XVIII ${ }^{\mathrm{e}}$ siècle. L'aspect introspectif de ses textes et la représentation de soi procèdent de Jean-Jacques Rousseau et de Joseph Joubert, son goût du calembour nous rappelle que le genre a pris son essor avec le marquis de Bièvre, tandis que ses saynètes grivoises évoquent la satire des vaudevilles ${ }^{57}$. Loin de réduire son intérêt ou d'amoindrir son originalité, rapprocher Lequeu des ouvres, des hommes ou des idées qui lui sont contemporaines, permet de mieux le situer et de mieux le comprendre, en dépassant nos préjugés et nos préconceptions.

" S'il y a histoire, de quoi est-ce l'histoire ? " écrivait Hubert Damisch dans L'Origine de la perspective, interrogeant les historiens de l'art " traditionnels" sur leur rapport à l'art ${ }^{58}$. Aujourd'hui, l'histoire de l'art s'intéresse moins à l'art qu'aux artistes, à la création, à l'existence et à la réception des œuvres d'art. Cela ne l'empêche pas de s'attacher aux phénomènes de réception et par conséquent de traverser des périodes qui ont enrichi notre compréhension des œeuvres. Mais son objectif n'est pas de rechercher des prophéties ou des mystères dans le regard individuel de l'observateur. Si cet observateur est un artiste, il convient de savoir ce qu'il en fait dans sa propre production. Si cet observateur est un écrivain, un philosophe ou
8. Jean Jacques Lequeu, "Âge nubile », Paris, $\mathrm{BnF}$, département des Estampes et de la

Photographie, RESERVE BOITE FOL-AE-15 (1) 
9. Jean Jacques Lequeu,

" Il est libre», Paris, BnF, EST RESERVE HA-80 (B, 7).

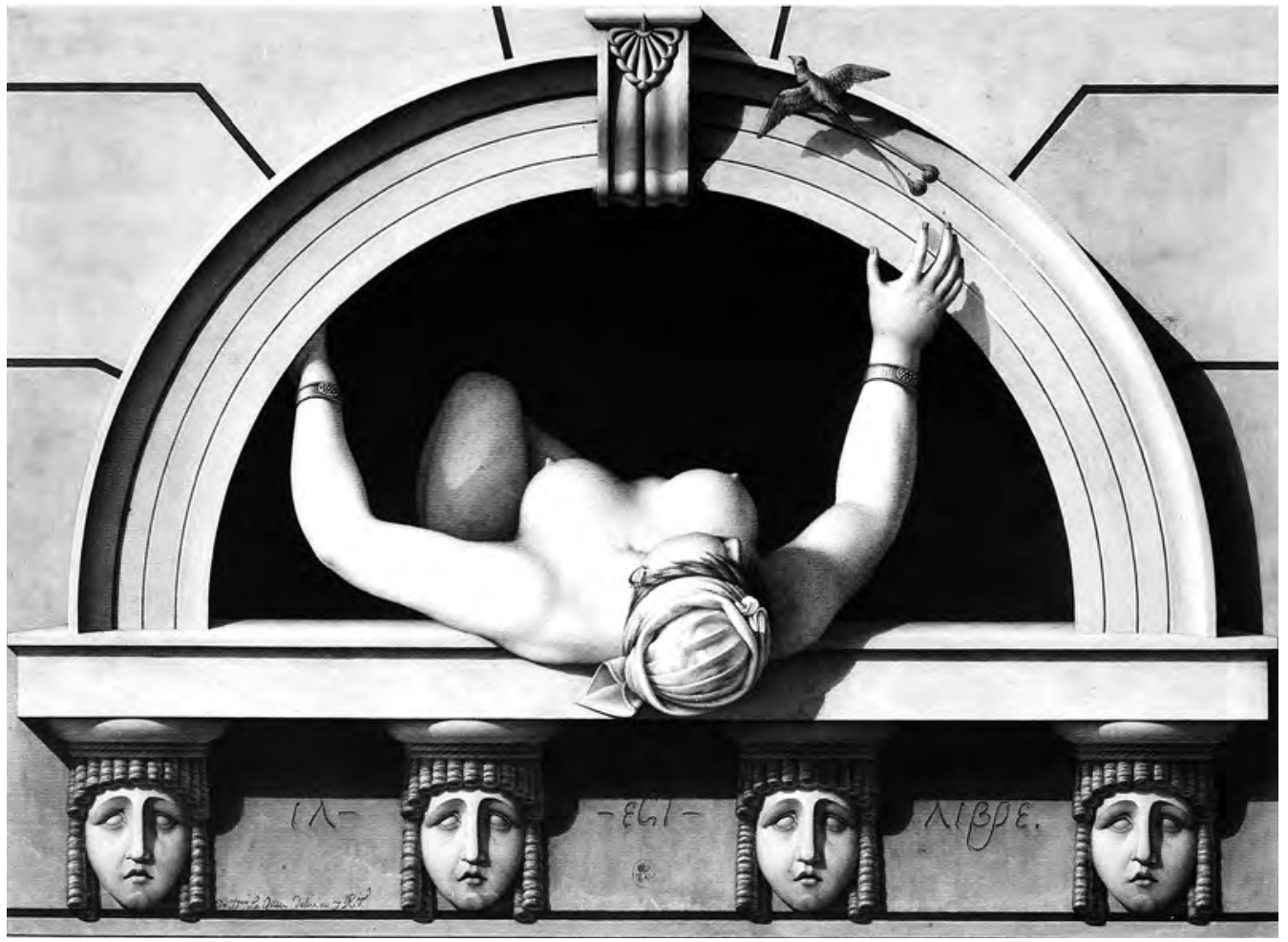

un historien de l'art, il importe de savoir ce qu'il a apporté à la compréhension de l'œuvre dans l'histoire de cette ouvre. Il ne s'agit pas de phénoménologie de la perception, mais de réception et d'apport de cette réception à la connaissance et à la compréhension.

Ces deux histoires de l'art coexistent, l'une plus ancienne que l'autre, l'une et l'autre connaissant des renouvellements et partageant certaines ouvertures disciplinaires - l'anthropologie en particulier. S'intéresser à l'action ou à l'effet de l'œuvre sur notre présent pour le replacer dans l'histoire peut avoir pour conséquence de restreindre l'observation à l'œuvre seule, à ignorer ce qui la lie aux autres productions qui lui sont contemporaines. C'est assurément ce qui fait l'intérêt de l'approche de Duboÿ sur Lequeu, mais qui implique aussi qu'elle doive elle-même, à trente ans de distance, être envisagée comme un objet historique selon une démarche accompagnant sa révision : si elle a eu le mérite d'amplifier la redécouverte de Lequeu, aujourd'hui, elle nous en apprend plus sur les années 1970-1980 que sur celles de Lequeu. Elle paraît désormais restreindre la connaissance de cette ouvre à certains questionnements empruntés au contexte historique de sa propre production. Être anachronique, c'est courir le risque de ne s'intéresser qu'aux artistes ou à celles de leurs œuvres qui parlent à notre temps, c'est ne pas voir ceux qui ne disent presque plus rien à nos regards et à nos idées. C'est limiter la production à des œuvres érigées au rang de paradigme d'une conception essentialiste de l'art. Restreindre ainsi le champ des œuvres étudiées, c'est paradoxalement limiter leur pouvoir anachronique, celui qui fait qu'elles existent grâce à tous ceux qui les conservent, les documentent, les exposent et les commentent bien au-delà de la mort de leur auteur.

Être anachronique, c'est paradoxalement être dans l'histoire car cette interprétation en a bien une, désormais. L'anachronisme est enraciné dans une conception de l'histoire qui appartient à une époque, car chaque époque à son "régime d'historicité59 ". Si tel n'était 
pas le cas, l'histoire de l'art " anachronique " devrait se contenter de répéter incessamment les mêmes vérités intemporelles, voire présentistes, en se référant à un XXe siècle qui, pourtant, est entré dans l'histoire. Être anachronique au non d'une nécessaire subjectivité dans l'appréhension du passé, n'est-ce pas s'exonérer à trop bon compte des promesses et des défis de l'altérité ? Lequeu, parce qu'il gravite dans un espace incertain entre le monde artisanal dont il est issu et celui des élites culturelles auquel il ne parvient pas à s'intégrer, ne nous incite-t-il pas, davantage encore qu'un artiste ayant pignon sur rue, à la prudence dans la réduction de la distance entre lui et nous ${ }^{60}$ ? Si le passé est un pays étranger, suivant la formule de David Lowenthal ${ }^{61}$, l'anachronisme ne court-il pas le risque de se réduire, en fin de compte, à un ethnocentrisme du contemporain ? 


\section{Notes}

Les auteurs souhaitent exprimer leur gratitude à tous ceux qui leur ont fait part de leurs réflexions et tout particulièrement à Ségolène Le Men pour ses précieuses suggestions.

1. Le livre et l'imprimé ont suscité un très grand nombre de travaux, couvrant toutes les périodes et proposant parfois un éclairage nouveau sur des figures bien connues, comme l'a fait Catherine de Smet avec Le Corbusier : Catherine de Smet, Vers une architecture du livre. Le Corbusier : édition et mise en page, Zurich, Lars Müller, 2007. Pour une synthèse des productions récentes dans ce domaine on pourra se reporter à Richard Wittman, " Print Culture and French Architecture in the Eighteenth and Nineteenth Centuries : a Survey of Recent Scholarship ", dans Perspective : actualité en histoire de l'art, no 1-2015, p. 113-132 [en ligne, URL : https://journals.openedition.org/ perspective/5806 ; DOI : 10.4000/perspective.5806 (consulté le 8 juin 2018)].

Dans le domaine du dessin, la récente exposition des Archives nationales, Dessiner pour bâtir. Le métier d'architecte au XVII' siècle (13 décembre 2017 au 12 mars 2018), représente un tournant pour une période longtemps dominée par les études monographiques centrées sur des figures d'architectes royaux : Alexandre Gady, Alexandre Cojannot (dir.), Dessiner pour bâtir. Le métier d'architecte au XVII siècle, Paris, Éditions du passage, 2017. Enfin, un intérêt pour des personnalités mineures ou marginales s'est manifesté ces dernières années à propos de Jules Bourgoin, ou de François Cointeraux : Maryse Bideault, Estelle Thibault et Mercedes Volait (dir.), De l'Orient à la mathématique de l'ornement: Jules Bourgoin, 1838-1908, Paris, CNRS, 2015 ; Maryse Bideault et alii, Jules Bourgoin (1838-1908). L'obsession $d u$ trait, cat. exp. (Paris, Institut national d'histoire de l'art, salle Roberto Longhi, 2012-2013), Paris, INHA, 2012 [en ligne, URL : http://inha.revues.org/4569 (consulté le 8 Juin 2018)] ; Laurent Baridon, Jean-Philippe Garric et Gilbert Richaud (dir.), Les leçons de la terre. François Cointeraux (1740-1830), professeur d'architecture rurale, Paris, INHA / Éditions des Cendres, 2016.

2. Jean Jacques Lequeu (1757-1826). Bâtisseur de fantasmes (Paris, Petit Palais, 11 décembre 2018 - 31 mars 2019). Le commissariat scientifique de l'exposition est assuré par Laurent Baridon, Jean-Philippe
Garric et Martial Guédron, avec des contributions de Corinne Le Bitouzé, Elisa Boeri, Valérie Nègre et Joëlle Raineau ; catalogue : Laurent Baridon, JeanPhilippe Garric et Martial Guédron (dir.), Jean Jacques Lequeu (1757-1826). Bâtisseur de fantasmes, cat. exp. (Paris, Petit Palais, 2018-2019), Paris, BnF / Norma, à paraître en décembre 2018 .

3. Elisa Boeri, Jean Jacques Lequeu : un atlas des mémoires. Histoire de l'Architecture civile, Paris, Éditions des Cendres, 2018 , à paraître ; Laurent Baridon, JeanPhilippe Garric et Martial Guédron, Jean Jacques Lequeu. Lexique, Paris, Éditions B2, 2018 , à paraître ; Laurent Baridon et Jean-Philippe Garric, " Incroyables et merveilleuses, les fabriques de parc de Jean Jacques Lequeu ", dans Revue de la $B n F$, vol. $2018 / 2$ (n $\mathrm{n}^{\circ}$ 5), p. 146-156.

4. Jean Charles Krafft, Plans, coupes et élévations des diverses productions de l'art de la charpente exécutées tant en France que dans les pays étrangers, Paris, Levrault, [1802]1805 ; Recueil d'architecture civile, contenant les plans, coupes et élévations des châteaux, maisons de campagne, et habitations rurales situées aux environs de Paris et dans les départements voisins, Paris, Bance, [1812].

\section{BOUCHOT, 1895.}

6. François Benoit, L'art français sous la Révolution et l'Empire. Les doctrines, les idées, les genres, Paris, L.-Henry May, 1897, p. 267.

7. Cité par DuBơ̈, 1987, p. 66.

8. "His weird fantasies reveal much of his era to one who is interested in the development of artistic ideas rather than in practical Improvement. Though Lequeu, like all Romanticists, looked back to remote times and remote regions, he was nonetheless a forerunner of a significant current in the early twentieth century. Here and there similar unrest and similar incertitude inspired strange performances; here and there expressiveness counted more than formality; then, as new, great and dignified works emerged from the turmoil. It is not my intention to imply any direct connection between 1800 and 1900; I am concerned only with the continuity of ideas. " KAUFMANN, 1952, p. 558. Les citations de cet ouvrage ont été traduites par les auteurs de l'article.

9. PAYOT, 1990, p. 40.

10. "I believe that not a personal condition, but the general unrest of the period must account for his production in the first place. Lequeu's dream-architecture marks the end of the period at the beginning of which stand the architectural dreams of Le Geay. Though Lequeu wandered beyond the regular bounds, his fantasies are more than extravaganzas. They are works of art in which we recognize the man, and through which we apprehend the period " (KAUFMANN, 1952, p. 544).

11. "Of the three, Boullee represents primarily the struggle for new forms; Ledoux, the search for a new order of the constituents; Lequeu the tragic ultimate stage of the revolutionary movement despair, resignation, and return to the past " (KAUFMANN, 1952, p. 435).

12. RosenAU, 1949, p. 111-116.

13. ROSENAU, 1950, p. 264-267.

14. Antonio Brucculeri (dir.), Louis Hautecour et la tradition classique, Paris, INHA, 2008 [en ligne, URL : http://journals. openedition.org/inha/2920 (consulté le 11 mars 2018)].

15. Louis Hautecour, Histoire de l'architecture classique en France, I. La formation de l'idéal classique, Paris, Auguste Picard, 1943, p. VII.

16. Louis Hautecour, Histoire de l'architecture classique en France, V. Révolution et Empire, 1792-1815, Paris, A. et J. Picard et Cie, 1953, p. 91.

17. Georg Germann, Vitruve et le vitruvianisme : introduction à l'histoire de la théorie architecturale (1987), Jacques Gubler (trad. fra.), Lausanne, PPUR, 1991, 6 ${ }^{\mathrm{e}}$ part. "Déclin du vitruvianisme ".

18. Jacques Guillerme consacre une étude exclusivement aux représentations de sexes féminins (GUILLERME, 1979, p. 138150).

19. "A mixture of incoherent and incongruous features, the Rendez-vous tells much of the period which struggled for innovation, and again and again turned to the past, haunted by weird dreams " (KAUFMANN, 1952, p. 558).

20. Thomas McEvilley, "Histoire de l'art ou histoire sainte ", dans Les Cahiers du Musée national d'art moderne, $\mathrm{n}^{\circ} 22$, décembre 1987, p. 125.

21. DUво Ÿ, 1976, p. 14-15.

22. Johann Rudolf Füssli, Johann Heinrich Füssli, Allgemeines Künstlerlexicon, oder: Kurze Nachricht von dem Leben und den Werken der Mahler, Bildhauer, Baumeister, Kupferstecher, Kunstgiesser, Stahlschneider [et]c. [et]c. Nebst angehängten Verzeichnissen der 
Lehrmeister und Schüler; auch der Bildnisse, der in diesem Lexicon enthaltenen Künstler, 6. vol., Zürich, Orell, 1806-1821, p. 695.

23. Benoit, 1897, cité n. 6, p. 267.

24. Odenthal, 1989, p. 39.

25. L'expression est employée par Breton en 1947 à propos d'artistes "présurréalistes " comme Bosch, Arcimboldo, Blake, Rousseau, Carroll. Le groupe surréaliste prépare alors l'Exposition internationale du Surréalisme qui doit avoir lieu à la Galerie Maeght de juillet à septembre 1947 ; voir André Breton, Euvres complètes, Marguerite Bonnet, avec la collaboration de Philippe Bernier, MarieClaire Dumas, Étienne-Alain Hubert et José Pierre (éd.), Paris, Gallimard (Bibliothèque de la Pléiade), t. 3, 1999, p. 1367.

26. " Pour moi, la plus forte est celle qui présente le degré d'arbitraire le plus élevé, je ne le cache pas; celle qu'on met le plus longtemps à traduire en langage pratique, soit qu'elle recèle une dose énorme de contradiction apparente, soit que l'un de ses termes en soit curieusement dérobé, soit que s'annonçant sensationnelle, elle ait l'air de se dénouer faiblement (qu'elle ferme brusquement l'angle de son compas), soit qu'elle tire d'ellemême une justification formelle dérisoire, soit qu'elle soit d'ordre hallucinatoire, soit qu'elle prête très naturellement à l'abstrait le masque du concret, ou inversement, soit qu'elle implique la négation de quelque propriété physique élémentaire, soit qu'elle déchaîne le rire " (André Breton, Manifeste du surréalisme, dans Breton, (1924) 1988, cité n. 25, t. 1, p. 338-339).

27. Laure Garcin, J. J. Grandville, révolutionnaire et précurseur de l'art du mouvement, Paris, E. Losfeld, 1970.

28. Laure Garcin, "Grandville, visionnaire, surréaliste, expressionniste ", dans Gazette des Beaux-Arts, t. XXXIV, 1948, p. 446.

29. Robert Venturi, Denise Scott Brown, Steven Izenour, L'enseignement de Las Vegas ou le symbolisme oublié de la forme architecturale, Sprimont, Mardaga, Architectures+Recherches, 1995, p. 17 [éd. orig. : Learning from Las Vegas, Cambridge, Mass., the MIT Press, 1972].

30. "There is an Egyptian House, a Chinese House, a Turkish House, a Little Synagogue, a Persian Porch, a Persian Sanctuary, a Hen House, with Oriental motifs, the Winepress, and the Pigeon-House, mixtures of Oriental and geometric shapes. There are several gothicizing projects, such as the Dairy, and the front of the Temple of Isis. ", KAUFMANN, 1952, p. 551.

31. GUILLERME, 1965, p. 154.

32. Voir par exemple David Kolb, Postmodern Sophistications. Philosophy, Architecture, and Tradition, Chicago, University of Chicago Press, 1990, p. 141-145.

33. GUILLERME, 1974, p. 180.

34. GUILLERME, 1979, p. 148.

35. DUвоŸ, 1976, p. 13-18.

36. Marcel Duchamp, Duchamp du signe, Michel Sanouillet (éd.), Paris, Flammarion, 1975.

37. Herbert Moldering, Duchamp traversé - Essais 1975-2012, Dijon, Les Presses du réel, 2014, p. 13-15.

38. L'édition américaine est parue en 1986 (DuвоŸ, 1986) ; voir en particulier le chapitre intitulé « Un Architecte en démolition " (DUвӧ̈, 1987).

39. Jean Baudrillard, Jean Nouvel, Les Objets singuliers : architecture et philosophie, Paris, Calmann-Lévy, 2000, p. 40-41.

40. François Cusset, French Theory. Foucault, Derrida, Deleuze o Cie et les mutations de la vie intellectuelle aux États-Unis, Paris, La Découverte, 2003, p. 257.

41. Jean-François Lyotard, La Condition postmoderne, Paris, Les Éditions de Minuit, 1979, p. 63 ; idem, Le postmodernisme expliqué aux enfants, Paris, Galilée, 1988, p. 38-41.

42. Fredric Jameson, Le Postmodernisme ou la logique culturelle du capitalisme tardif, $\mathrm{Pa}$ ris, Beaux-arts de Paris les éditions, 2007, p. 57-58.

43. Kolb, 1990, cité n. 32, p. 88-90.

44. David Harvey, The Condition of Posmodernity. An Enquiry into the Origins of Cultural Change, Cambridge, Mass. / Oxford, Blackwell Publishers, 1990, p. 54.

45. Jean-François Lyotard, "Réponse à la question : qu'est-ce que le postmoderne ?", dans Critique, no 419 , avril 1982, p. 357-367.

46. Non seulement le "photoactionnisme " pratiqué dans la constellation surréaliste commençait à être mieux étudié depuis la fin des années 1970, mais il servait aussi d'amorce à une nouvelle génération d'artistes, comme Cindy Sherman avec sa série Untitled Film Still (1978).

47. Voir « Histoire littéraire et science de la littérature ", dans Essais I, 1922-1934, Maurice Gandillac (trad. fra.), Paris, Denoël/Gonthier, 1983, p. 148.
48. Cité par Danièle Cohn (dir.), $Y$ voir mieux, $y$ regarder de plus près : Autour d'Hubert Damisch, actes du colloque (Rome, Villa Médicis - Académie de France à Rome, 1999), Paris, Éditions Rue d'Ulm, 2003, p. 11.

49. Hubert Damisch, Giovanni Careri et Bernard Vouilloux, " Hors cadre : entretien avec Hubert Damisch ", dans Perspective. Période moderne, $\mathrm{n}^{\circ}$ 1-2013, p. 11-23.

50. Aby Warburg, Essais florentins, Sybille Muller (trad. fra.), Éveline Pinto (présentation), Paris, Klincksieck, 1990, p. 255.

51. Walter Benjamin, « Paralipomènes et variantes des "thèses sur le concept d'histoire" ", J.-M. Monnoyer (trad. fra.), dans Écrits français, Paris, Gallimard, 1991, p. 180. Voir Didi-HUBERMAN, 2000, p. 93.

52. C'était, par exemple, un des enjeux stimulants des deux expositions organisées par le département des Arts graphiques du musée du Louvre à l'initiative de Régis Michel au début des années 2000, Posséder et détruire: stratégies sexuelles dans l'art d'Occident (2000) et La Peinture comme crime ou la part maudite de la modernité (2001).

53. Georges Louis Le Rouge, Jardins anglochinois à la mode, Paris, l'auteur, 1775 1789. Voir Véronique Royet et al., Georges Louis Le Rouge. Jardins anglo-chinois, Paris, Bibliothèque nationale de France, 2004 ; Jean Charles Krafft, Plans des plus beaux jardins pittoresques de France, d'Angleterre et d'Allemagne, Paris, De l'imprimerie de Levrault, 1808-1810.

54. Louis Sébastien Mercier, Tableau de Paris, Jean-Claude Bonnet (éd.), Paris, Mercure de France, 1994, t. 1, p. 1323.

55. Thomas Laqueur, La fabrique du sexe. Essai sur le corps et le genre en Occident, Paris, Gallimard (NRF-Essais), 1992, p. 191.

56. Jacques Fabien Gautier d'Agoty, Exposition anatomique des maux vénériens, sur les parties de l'homme et de la femme, et les remèdes les plus usités [...], Paris, J.-B. Brunet / Demonville, 1773.

57. François Georges Maréchal de Bièvre, Calembours et autres jeux sur les mots d'esprit, Antoine de Baecque (éd.), Paris, Payot, 2000.

58. Hubert Damisch, L'origine de la perspective, Paris, Flammarion (Champs), 1993, p. 23.

59. François Hartog, Régimes d'historicité, Présentisme et expériences du temps, Paris, Éditions du Seuil, 2003. 
60. On pense à la remarque de Carlo Ginsburg, à propos du meunier Menocchio, selon laquelle " La mauvaise conscience du colonialisme rejoint ainsi la mauvaise conscience de l'oppression de classe "; Carlo Ginsburg, Le Fromage et les vers, Paris, Aubier, 1980, p. 8.

61. David Lowenthal, The Past is a Foreign Country, Cambridge, Cambridge University Press, 1985.

\section{Bibliographie}

- Bouchot, 1895 : Henri Bouchot, Le Cabinet des Estampes de la Bibliothèque nationale : guide du lecteur et du visiteur, catalogue général et raisonné des collections qui y sont conservées, Paris, Dentu, 1895, vol. 1, p. 14, 92, 118, 155, 187, 210.

- Didi-Huberman, 2000 : Georges DidiHuberman, Devant le temps, Histoire de l'art et anachronisme des images, Paris, Les Éditions de Minuit, 2000.

- DuвоŸ, 1976 : Philippe Duboy, « J. J. Lequeu (1757- ?), Duchamp ", dans XXe siècle, ${ }^{\circ} 47,1976$, p. 13-18.

- DUвӧ̈, 1986 : Philippe Duboÿ, Lequeu: an Architectural Enigma, Cambridge, Massachusetts, the MIT Press, 1986.

- Duвơ̈, 1987 : Philippe Duboÿ, JeanJacques Lequeu, une énigme, Paris, Hazan, 1987.

- GUILLERME, 1965 : Jacques Guillerme, "Lequeu et l'invention du mauvais goût ", dans Gazette des Beaux-Arts, LXVI, 1965, p. 153-166.

- GUILLERME, 1966 : Jacques Guillerme, Marianne Boegner, "Thèmes, partis et formes chez l'architecte Lequeu ", dans L'art et la psychopathologie, numéro spécial trimestriel de La vie médicale, Noël 1966, $n^{\circ} 47,1966$, p. 67-82.

- GUILLERME, 1974 : Jacques Guillerme, " Lequeu entre l'irrégulier et l'éclectique ", dans Dix-huitième siècle, $\mathrm{n}^{\circ}$ 6, 1974, p. 167-180.

- GuILLERME, 1979: [Jacques Guillerme] Jean-Jacques Marty-L'Herme, "Les cas de Jean-Jacques Lequeu ", dans Macula, $\mathrm{n}^{\circ}$ 5/6, 1979, p. 138-149.

- Kaufmann, 1949 : Emil Kaufmann, "Jean-Jacques Lequeu ", dans The Art Bulletin, $\mathrm{n}^{\circ}$ 1-4, mars 1949, p. 130-135.

- KaUfmanN, 1952 : Emil Kaufmann, "Three Revolutionary Architects, Boullée, Ledoux and Lequeu ", dans Transactions of the American Philosophical Society, vol. 42, part. 3, 1952 [trad. fra. : Trois architectes révolutionnaires: Boullée, Ledoux, Lequeu, Gilbert Erouart, Georges Teyssot (introduction et notes), Paris, les éditions de la SADG, 1978].

- NAGEL, Wood, 2010 : Alexander Nagel, Christopher S. Wood, Anachronic Renaissance, New York, Zone books, 2010 [Renaissance anachroniste, Françoise Jaouën (trad. fra.), Dijon, Les Presses du réel, 2015].

- ODENTHAL, 1986 : Johannes Odenthal, Lequeu. Imaginäre Architekur, Frankfort
I New York, Qumran im Campus-Verl, 1986.

- ODENTHAL, 1989: Johannes Odenthal, "Lequeu's Architecture civile and the Kosmos of Alexander von Humboldt ", dans Daidalos, $\mathrm{n}^{\circ}$ 34, 15 décembre 1989, p. 30-41.

- PAYOT, 1990 : Daniel Payot, Anachronies de l'auvre d'art, Paris, Galilée, 1990.

- Rosenau, 1949 : Helen Rosenau, « Architecture and the French Revolution: Jean-Jacques Lequeu ", dans Architectural Review, vol. 106, nº 632, août 1949, p. 111-116.

- Rosenau, 1950 : Helen Rosenau, " Postscript on Lequeu ", dans Architectural Review, vol. 108, nº 646, octobre 1950 , p. 264-267. 